\title{
Analysis of 3D time-dependent acoustic problems via a generic BE substructuring algorithm based on iterative solvers
}

\author{
F.C. Araújo*, D.R. Alberto, C. Dors ${ }^{1}$ \\ Department of Civil Engineering/UFOP, CEP 35400-000 Ouro Preto-MG, Brazil
}

Received 20 May 2002; revised 25 November 2002; accepted 27 November 2002

\begin{abstract}
In this paper, a generic $\mathrm{BE} / \mathrm{BE}$ coupling algorithm based on iterative solvers is applied to solve 3D time-dependent acoustic problems. As regards the treatment of the time-dependence, a direct time-marching scheme is considered. Several types of boundary elements and cells are available in the code for the spatial description of the involved variables. Concerning the BE/BE coupling technique, its chief idea is to completely avoid storing and manipulating the zero blocks appearing in the coupled system by the use of iterative solvers. The global system matrix is not explicitly assembled; instead the algebraic subsystems (associated with the substructures of the model) are manipulated as they were independent of each other. An insight into the coupling strategy and the used iterative solver (Jacobi-preconditioned bi-conjugate gradient method) is given. Analyses of sound barriers are carried out for verifying the performance of the respective computational code modules.
\end{abstract}

(C) 2003 Elsevier Ltd. All rights reserved.

Keywords: 3D Acoustics; Transient analyses; BE/BE coupling algorithms; Iterative schemes

\section{Introduction}

Sound waves take place when pressure disturbances propagate through a compressible fluid. Their investigation is necessary in a great many technical problems as, e.g. in the noise reduction near highways and in airports, prediction of sound fields in auditoria, noise control in industrial installations, design of vehicles, and several other situations associated with the comfort of human beings [1].

Important works concerning transient analyses in 2D acoustics by boundary element models were presented by Mansur and Brebbia [2,3] and Mansur [4]. After these first works, Dohner et al. [5], Meise [6], Antes [7], and Araújo et al. [8] published more advanced works related to $3 \mathrm{D} \mathrm{BE}$ acoustic analyses in the time-domain. More recently, Antes and Baaran [9] applied the direct BEM to

\footnotetext{
* Corresponding author. Tel.: +55-31-3559-1547; fax: +55-31-35591548 .

E-mail addresses: fcelio@em.ufop.br (F.C. Araújo),denival@em.ufop. br (D.R. Alberto), dors@coc.ufrj.br (C. Dors).

${ }^{1}$ Tel.: + 55-21-2562-7196.
}

analyze 3D noise radiation caused by independently moving surfaces. In this case, a time-domain $\mathrm{BE}$ formulation is indeed necessary. To describe the convective effects associated with the moving boundaries, they used the approach proposed by Gennaretti and Morino [10].

In this paper, the coupling strategy previously presented by Araújo et al. [11], which proved to be efficient for non-homogeneous frequency-dependent problems, is applied to solve general 3D transient acoustic problems. As a boundary element formulation is involved, the procedure is quite suitable for analyzing exterior domain problems (infinite domains). On the other hand, the substructuring option (BE/BE coupling) is necessary for modeling thin elements, as, e.g. sound barriers, by means of direct standard BEM formulations.

To treat two-dimensional time-harmonic acoustic problems defined in unbounded different media, Cremers et al. [12] developed a special multi-domain BEM, in which infinite boundary elements are used to model boundaries and interfaces of infinite extent. Though not 
necessary [13], this approach describes open domains more correctly. Other characteristic of the formulation presented by Cremers et al. [12] is that as a consequence of the chain assembly of the subdomains, i.e. subdomain $j$ is coupled only with subdomains $j-1$ and $j+1$, the global non-symmetric matrix is banded. It is unfortunately not always possible to work with chain subdomain models, and, as a matter of fact, for modeling more general regions with use of substructures, more complicated sparse matrices are obtained as well. Optimized algorithms as, for instance, skyline and frontal ones are then necessary for solving efficiently the coupled system [14]. In this paper, the general and ideally optimized BE/ BE algorithm proposed in Ref. [11] is applied to solve time-domain acoustic problems.

As in the case of the design of sound barriers both thin elements and open domains are present, the algorithm proposed in this paper may be a very promising one. There are nowadays boundary element formulations based on the hypersingular integral equation, the so-called dual boundary element method, that has been successfully applied to simulate nonthickness bodies, as for instance sound barriers [15-18]. As a Cauchy principal value and a Hadamard finite part are present in the traction integral equation, special algorithms for their efficient evaluation $[19,20]$ must be taken into account. An advantage of dual boundary element formulations is that no additional interfaces for modeling non-thickness barriers are necessary; this improves the precision of the results.

As regards the modeling of open domain with flat ground, efficient boundary element formulations based on the use of half-space Green's functions [16,21,22] may also be derived; in this way one avoids discretizing the ground. In Ref. [23], Green's functions that simulate the presence of tall buildings in the vicinity of sound barriers are also presented.

However, even in the cases above, in which dual BE formulations or Green's functions for flat half-spaces are used, BE/BE coupling strategies may be inevitable; for instance, when non-homogeneous regions or large models have to be analyzed. In the latter case, substructuring techniques may be very useful for carrying out parallelized analyses.

In effect, substructuring strategies constitute a very important and general technique in computer mechanics, not exclusively restricted to $\mathrm{BE}$ formulations, but that considerably extend their range of applications. In previous works [14,24,25], either non-condensed or condensed strategies based on the use of direct solvers are reported.

To analyze real engineering problems, usually large, efficient procedures that reduce the storage area to a minimum and also be fast, must be idealized. In effect, these two topics are addressed in this paper, then iterative solvers make it possible to reduce the storage area to a minimum, as the null coefficients of the sparse coupled system are not stored, and moreover may be much faster than the direct ones for large systems. Indeed, in the 90s, the iterative methods gained more and more acceptance in real-life industrial applications. Important recent books covering the use of iterative solvers in engineering were published by Axelsson [26], Hack-busch [27], and Saad [28]. In the particular case of BE systems of equations, successful applications of iterative methods have already been done by Mansur et al. [29], Barra et al. [30], Prasad et al. [31], Hribersek and Skerget [32], Leung and Walker [33], Skerget et al. [34], and Valente and Pina [35]. In these works, Krylov subspace methods that apply to general unsymmetric systems of equations are considered; mainly preconditioned versions of the BiCG, the GMRES, and hybrid BiCG methods, obtained by combining BiCG methods with other Krylov iterative methods [36], were incorporated into $\mathrm{BE}$ algorithms. In this paper, the Jacobipreconditioned BiCG is the only solver used in the BE/ BE coupling strategy.

As for linear transient analyses the system matrix remains unchanged during the whole analysis time, one may think that a LU-based direct solver should be the most attractive alternative. In fact, the matrix decomposition for a really large problem is a very hard computational task, so that iterative methods, even for linear transient problems, may be an interesting option. In this paper, one adopts as initial guess for calculating the current system solution that one of the last time step.

The analysis of sound barriers is considered for verifying the performance of the respective computer code modules. Important parameters for estimating the efficiency of the algorithms, as required CPU times, number of iterations, memory used, and response accuracy are discussed in the results of the paper.

\section{Time-dependent boundary integral formulation}

Small-amplitude sound waves are described by means of the linearized wave equation

$u_{, i i}-\left(\frac{1}{c^{2}}\right) u_{, t t}=-\gamma(\mathbf{x}, t)$,

where $u(\mathbf{x}, t)$ can be either the velocity potential or the acoustic pressure, and $\gamma(\mathbf{x}, t)$ is a general volume source of acoustic energy $[1,8]$. Eq. (1) is to be solved under certain boundary conditions (prescribed acoustic pressure, velocity or acoustic impedance), and also under known initial values.

By using a general weighted residual statement [4] or by using Laplace transforms [8], Eq. (1), defined in a region $\Omega$ with boundary $\Gamma$, can be converted into the following 
general boundary integral equation

$$
\begin{aligned}
& c(\xi) u(\xi, t)+\int_{\Gamma} \int_{0}^{t} p^{*}(\mathbf{x}, t ; \xi, \tau) u(\mathbf{x}, \tau) \mathrm{d} \tau \mathrm{d} \Gamma(\mathbf{x}) \\
& =\int_{\Gamma} \int_{0}^{t} u^{*}(\mathbf{x}, t ; \xi, \tau) p(\mathbf{x}, \tau) \mathrm{d} \tau \mathrm{d} \Gamma(\mathbf{x}) \\
& \quad+\int_{\Omega} \int_{0}^{t} u^{*}(\mathbf{x}, t ; \xi, \tau) \gamma(\mathbf{x}, \tau) \mathrm{d} \tau \mathrm{d} \Omega(\mathbf{x}) \\
& \quad+c^{-2} \int_{\Omega}\left\{v_{0}(\mathbf{x}) u^{*}(\mathbf{x}, t ; \xi, 0)\right. \\
& \left.\quad+u_{0}(\mathbf{x}) \dot{u}^{*}(\mathbf{x}, t ; \xi, 0)\right\} \mathrm{d} \Omega(\mathbf{x}),
\end{aligned}
$$

where $u^{*}$ and $p^{*}$ are, respectively, the fundamental solution and flux, given by

$u^{*}(x, t ; \xi, 0)=\frac{1}{4 \pi r} \delta\left(t-\frac{r}{c}\right)$

$p^{*}(\mathbf{x}, t ; \xi, 0)=-\frac{1}{4 \pi r^{2}}\left\{\delta\left(t-\frac{r}{c}\right)+\frac{r}{c} \dot{\delta}\left(t-\frac{r}{c}\right)\right\} \frac{\partial r}{\partial \mathbf{n}}$,

and the integral free term $c(\xi)$ is calculated by

$c(\xi)=1+\lim _{\varepsilon \rightarrow 0} \int_{\bar{\Gamma}_{\varepsilon}}{ }^{\mathrm{st}} p^{*}(\mathbf{x}, \xi) \mathrm{d} \Gamma(\mathbf{x})$,

${ }^{\text {st }} p^{*}(\mathbf{x}, \xi)$ representing the fundamental flux in $\Gamma$ for timeindependent potential problems. In Eq. (5), $\bar{\Gamma}_{\varepsilon}$ is a spherical surface centered at $\xi$, introduced for evaluating the improper integrals [8], and $c(\xi)$ corresponds exactly to the relationship

$c(\xi)=\frac{\Phi_{i}}{4 \pi}$,

where $\Phi_{i}$ is the inner solid angle at $\xi$ (Fig. 1).

Moreover, it should be noticed that the singular boundary integral in Eq. (2) associated with $p^{*}(\mathbf{x}, t ; \xi, \tau)$ exists only in the sense of the Cauchy principal value. In Araújo et al. [8],

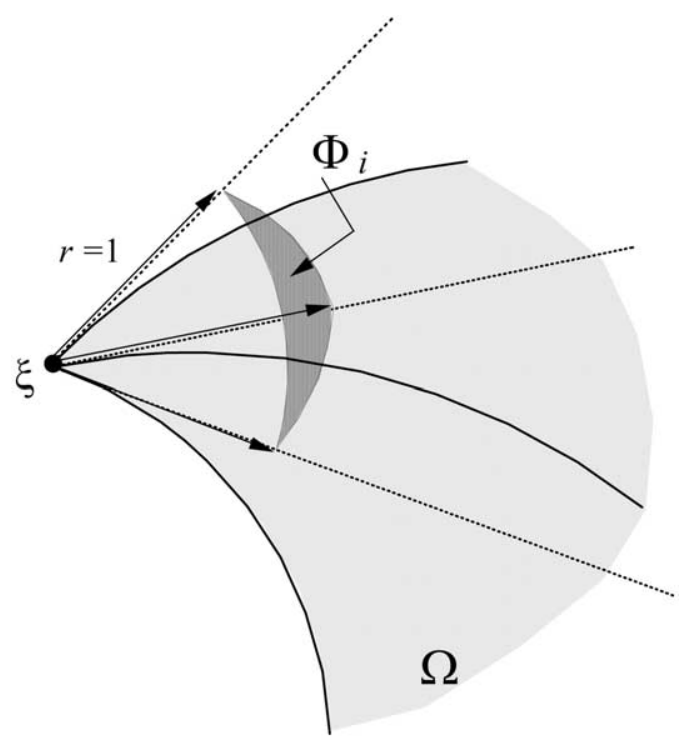

Fig. 1. Inner solid angle $\Phi_{i}$. a detailed derivation of the above boundary integral equation is provided.

In this paper, the description of the acoustic problem based on the excess acoustic pressure will be considered. One advantage of such a formulation is that the variables involved have physical meaning (pressures and particle velocities). Thus, as in the boundary integral formulation the function $p(\mathbf{x}, t)$ corresponds to normal derivatives of the acoustic pressure, this function does not directly represent the particle velocities. An additional transformation is needed to associate the normal derivative at $\mathbf{x}, p(\mathbf{x}, t)$, with the respective particle velocity. Namely, the following relationship, derived from the Euler's equation [1], must be considered

$p(\mathbf{x}, t)=-\rho_{0} \frac{\partial v_{n}(\mathbf{x}, t)}{\partial t}$

where $\rho_{0}$ is the equilibrium density of the fluid, and $v_{n}$ denotes the velocity component of its particles normal to the boundary $\Gamma$ at $(\mathbf{x}, t)$.

\section{The time-dependent algebraic system}

To derive the system of algebraic equations, the collocation method is used with respect to the space and time domains. Boundary elements are employed to approximate the boundary geometry and values, and integration cells, for evaluating the contributions of timedependent volume sources and initial conditions. The approximation of the field variables along the time is carried out with usual time interpolation functions (Fig. 2). Thus, Eq. (2) can be transformed into a time-dependent system of algebraic equations, whose solution supplies the boundary unknowns $u$ and $p$ at the current time. Different types of boundary elements and integration cells are available in the computer code; namely, quadrangular and triangular, linear and quadratic boundary elements, and parallelepipedic linear and quadratic cells have already been implemented. Concerning the discretization of timedomain, a formulation that takes into account interpolation functions of a generic order (constant, linear, quadratic, etc.)

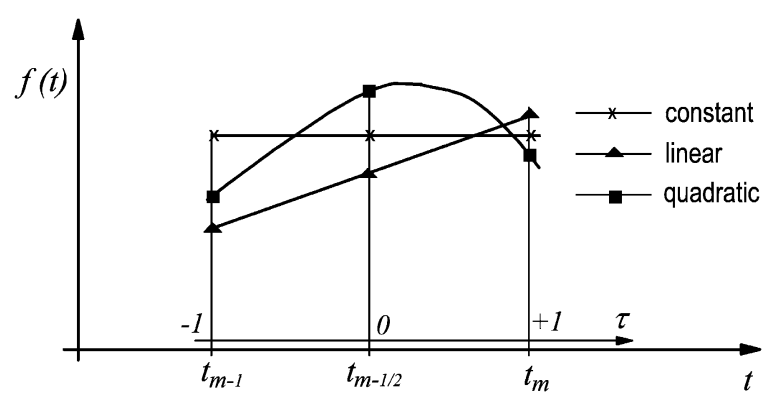

Fig. 2. Time interpolation functions. 
is provided below; yet the case of constant and linear time interpolation functions will be particularly emphasized.

To obtain the time-discretized version of Eq. (2), the initial conditions will be temporarily neglected, then they are actually not important for deriving the time-marching scheme. Thus, by generically approximating $u(\mathbf{x}, \tau), p(\mathbf{x}, \tau)$ and $\gamma(\mathbf{x}, \tau)$ within the $m$ th time step by

$u^{(m)}(\mathbf{x}, \tau)=\sum_{\alpha=1}^{n_{\alpha}} L_{\alpha}^{(m)}(\tau) u^{\alpha(m)}(\mathbf{x})$,

$p^{(m)}(\mathbf{x}, \tau)=\sum_{\beta=1}^{n_{\beta}} M_{\beta}^{(m)}(\tau) p^{\beta(m)}(\mathbf{x})$,

$\gamma^{(m)}(\mathbf{x}, \tau)=\sum_{\zeta=1}^{n_{\zeta}} N_{\zeta}^{(m)}(\tau) \gamma^{\zeta(m)}(\mathbf{x})$,

the following equation is found

$$
\begin{gathered}
c(\xi) u\left(\xi, t_{n}\right)+\sum_{m=1}^{\bar{n}} \sum_{\alpha=1}^{n_{\alpha}} \int_{\Gamma}{ }^{(\alpha)} P^{n m}(\mathbf{x} ; \xi) u^{\alpha(m)}(\mathbf{x}) \mathrm{d} \Gamma(\mathbf{x}) \\
=\sum_{m=1}^{\bar{n}} \sum_{\beta=1}^{n_{\beta}} \int_{\Gamma}{ }^{(\beta)} U^{n m}(\mathbf{x} ; \xi) p^{\beta(m)}(\mathbf{x}) \mathrm{d} \Gamma(\mathbf{x}) \\
+\sum_{m=1}^{\bar{n}} \sum_{\zeta=1}^{n_{\zeta}} \int_{\Omega}{ }^{(\zeta)} \bar{U}^{n m}(\mathbf{x} ; \xi) \gamma^{\zeta(m)}(\mathbf{x}) \mathrm{d} \Omega(\mathbf{x})
\end{gathered}
$$

with

$$
\begin{aligned}
{ }^{(\alpha)} P^{n m}(\mathbf{x} ; \xi) & =\int_{t_{m-1}}^{t_{m}} p^{*}\left(\mathbf{x}, t_{n} ; \xi, \tau\right) L_{\alpha}^{(m)}(\tau) \mathrm{d} \tau, \\
{ }^{(\beta)} U^{n m}(\mathbf{x} ; \xi) & =\int_{t_{m-1}}^{t_{m}} u^{*}\left(\mathbf{x}, t_{n} ; \xi, \tau\right) M_{\beta}^{(m)}(\tau) \mathrm{d} \tau, \\
{ }^{(\zeta)} \bar{U}^{n m}(\mathbf{x} ; \xi) & =\int_{t_{m-1}}^{t_{m}} u^{*}\left(\mathbf{x}, t_{n} ; \xi, \tau\right) N_{\zeta}^{(m)}(\tau) \mathrm{d} \tau .
\end{aligned}
$$

In Eqs. (8)-(14), $n$ indicates the current time point, $\bar{n}$ is the current time step, $n_{\alpha}, n_{\beta}$ and $n_{\zeta}$ are the number of (time) points used for the time interpolation of $u(\mathbf{x}, \tau)$, $p(\mathbf{x}, \tau)$ and $\gamma(\mathbf{x}, \tau)$, respectively, $u^{\alpha(m)}(\mathbf{x}), p^{\beta(m)}(\mathbf{x})$ and $\gamma^{\zeta(m)}(\mathbf{x})$ denote the values of the respective functions at the time points $\alpha, \beta$ and $\zeta$ of the $m$ th time step, $L_{\alpha}^{(m)}(\tau)$, $M_{\beta}^{(m)}(\tau)$ and $N_{\zeta}^{(m)}(\tau)$ are the adopted time interpolation functions, and the time integration limits in Eqs. (12)(14) are given by

$t_{m}= \begin{cases}m \Delta t, & \text { if } m<\bar{n}, \\ \bar{m} \Delta t, & \text { if } m=\bar{n},\end{cases}$

where

$\bar{m}= \begin{cases}m, & \text { if } n_{a} \leq 2, \\ {\left[(\bar{n}-1)+\frac{(a-1)}{\left(n_{a}-1\right)}\right],} & \text { if } n_{a} \geq 3,\end{cases}$

with $a=2, \ldots n_{a}, n_{a}=\max \left(n_{\alpha}, n_{\beta}\right)$. Note that the parameter $a$ indicates the local order of the discrete time $t_{n}$, necessary to make possible the evaluation of the problem response at all time points of the current time step.

After considering the time translation property of the acoustic fundamental solution [8], the time-integrated kernels ${ }^{(\alpha)} P^{n m},{ }^{(\beta)} U^{n m}$, and ${ }^{(\zeta)} \bar{U}^{n m}$ in Eqs. (12)-(14) can be reduced to integrations along the first time step only. Thus, at each time step, one has to calculate only the following time integrations

$$
\begin{aligned}
{ }^{(\alpha)} P^{(n)}(\mathbf{x} ; \xi) & =\int_{0}^{\bar{m}_{1} \Delta t} p^{*}\left(\mathbf{x}, t_{(n)} ; \xi, \tau\right) L_{\alpha}(\tau) \mathrm{d} \tau \\
{ }^{(\beta)} U^{(n)}(\mathbf{x} ; \xi) & =\int_{0}^{\bar{m}_{1} \Delta t} u^{*}\left(\mathbf{x}, t_{(n)} ; \xi, \tau\right) M_{\beta}(\tau) \mathrm{d} \tau \\
{ }^{(\zeta)} \bar{U}^{(n)}(\mathbf{x} ; \xi) & =\int_{0}^{\bar{m}_{1} \Delta t} u^{*}\left(\mathbf{x}, t_{(n)} ; \xi, \tau\right) N_{\zeta}(\tau) \mathrm{d} \tau
\end{aligned}
$$

with

$\bar{m}_{1}= \begin{cases}1, & \text { if } n_{a} \leq 2, \\ \frac{(a-1)}{\left(n_{a}-1\right)}, & \text { if } n_{a} \geq 3,\end{cases}$

$a=2, \ldots, n_{a}, n_{a}=\max \left(n_{\alpha}, n_{\beta}\right)$. The time integrations in Eqs. (17)-(19) are very simple and can be easily calculated in closed form. In Ref. [8], the results of the integrals in Eqs. (17)-(19) are furnished.

The above expressions are generic and may be used to obtain the system of algebraic equations associated with transient acoustic problems. Here, these expressions are however, particularized to the following time interpolations functions: the potentials are linearly interpolated $\left(n_{\alpha}=2\right.$ in Eq. (7)), the fluxes, constant- or linearly $\left(n_{\beta}=1\right.$ or $n_{\beta}=2$, respectively, in Eq. (8)), and the volume sources, constantly interpolated $\left(n_{\zeta}=1\right.$ in Eq. (9)). In this way, after the spatial discretization of the time-discretized boundary integral equation (11), one obtains

$$
\left\{\mathbf{C}+{ }^{(2)} \mathbf{H}^{(1)}\right\} \mathbf{u}^{(n)}={ }^{(2)} \mathbf{G}^{(1)} \mathbf{p}^{(n)}+\mathbf{r}^{(n)},
$$

where

$$
\begin{aligned}
\mathbf{r}^{(n)}= & { }^{(1)} \mathbf{G}^{(n)} \mathbf{p}^{(0)}+\sum_{m=n_{i}}^{n-1}\left\{{ }^{(1)} \mathbf{G}^{(n-m)}+{ }^{(2)} \mathbf{G}^{(n-m+1)}\right\} \mathbf{p}^{(m)} \\
& -\sum_{m=n_{i}}^{n-1}\left\{{ }^{(1)} \mathbf{H}^{(n-m)}+{ }^{(2)} \mathbf{H}^{(n-m+1)}\right\} \mathbf{u}^{(m)} \\
& +\sum_{m=n_{i}}^{n-1} \overline{\mathbf{G}}^{(n-m+1)} \mathbf{g}^{(m)}
\end{aligned}
$$

with

$n_{i}=\max \left(1, n-n_{\max }+1\right), \quad n_{\max }=\frac{r_{\max }}{c \Delta t}+2$.

Note that $n_{i}$ in Eq. (23) expresses the truncation of the time-marching scheme and is determined based on the 
existence interval of the fundamental kernels given by $(n-1) c \Delta t<r<n c \Delta t$ [8].

In case of existing initial values to be considered, their contributions will be stored in an additional vector on the right-hand side of the system of Eq. (21), which will be dependent on only of the current time step. This vector will be determined in the most general cases with the use of integration cells.

\section{The time-dependent BE/BE coupling}

In order to develop the generic substructuring algorithm, the system of Eq. (21) is written for each subregion of the coupled system. The following systems are available

$\mathbf{H}^{k} \mathbf{u}^{(n), k}=\mathbf{G}^{k} \mathbf{p}^{(n), k}+\mathbf{r}^{(n), k}, \quad k=1, \mathrm{~ns}$,

where ns is the number of subregions of the model. By organizing the system of Eq. (24), so that one obtains on its left-hand side the unknown boundary values and the unknown interface potentials, and on its right-hand side the known boundary values and the unknown interface normal fluxes, it results:

$\left[\begin{array}{ll}\mathbf{A}_{b}^{k} & \mathbf{H}_{i}^{k}\end{array}\right]\left\{\begin{array}{l}\mathbf{x}_{b}^{(n), k} \\ \mathbf{u}_{i}^{(n), k}\end{array}\right\}=\left[\begin{array}{ll}\mathbf{B}_{\bar{b}}^{k} & \mathbf{G}_{i}^{k}\end{array}\right]\left\{\begin{array}{l}\mathbf{y}_{\bar{b}}^{(n), k} \\ \mathbf{p}_{i}^{(n), k}\end{array}\right\}+\mathbf{r}^{(n), k}$,

where

$$
\begin{array}{ll}
\mathbf{A}_{b}^{k}=\left[\begin{array}{ll}
\mathbf{H}_{b(u)}^{k} & -\mathbf{G}_{b(p)}^{k}
\end{array}\right], & \mathbf{x}_{b}^{(n), k}=\left\{\begin{array}{c}
\mathbf{u}_{b}^{(n), k} \\
\mathbf{p}_{b}^{(n), k}
\end{array}\right\}, \\
\mathbf{B}_{\bar{b}}^{k}=\left[\begin{array}{ll}
-\mathbf{H}_{b(\bar{u})}^{k} & \mathbf{G}_{b(\bar{p})}^{k}
\end{array}\right], & \mathbf{y}_{\bar{b}}^{(n), k}=\left\{\begin{array}{c}
\overline{\mathbf{u}}_{b}^{(n), k} \\
\overline{\mathbf{p}}_{b}^{(n), k}
\end{array}\right\} .
\end{array}
$$

The subscripts above are defined as follows: $b(u)$ defines the boundary part of the $k$ th subregion with unknown potentials, $b(p)$ that one with unknown fluxes, and $i$ that one corresponding to the interface surfaces. $b(\bar{u})$ and $b(\bar{p})$ define, respectively, the boundary part with prescribed potential and normal flux.

As corresponding to the interface nodes of a given subregion $k$, both potentials and normal fluxes are unknowns, an additional number of equations will be necessary for making it possible to calculate all its unknown boundary values (including naturally its interface values). These equations are actually available in the equations of the other subdomains, so that if one assembles together the subsystems corresponding to all the substructures of the problem, a unique global system of equations with enough equations for determining the response of the problem in each subregion is obtained. In explicit form, this system is given by

$$
\begin{aligned}
& {\left[\begin{array}{cccccccc}
\mathbf{A}_{b}^{1} & \mathbf{0} & \cdots & \mathbf{0} & \mathbf{C}_{1}^{1} & \mathbf{C}_{2}^{1} & \cdots & \mathbf{C}_{s}^{1} \\
\mathbf{0} & \mathbf{A}_{b}^{2} & \cdots & \mathbf{0} & \mathbf{C}_{1}^{2} & \mathbf{C}_{2}^{2} & \cdots & \mathbf{C}_{s}^{2} \\
\vdots & \vdots & \ddots & \vdots & \vdots & \vdots & \ddots & \vdots \\
\mathbf{0} & \mathbf{0} & \cdots & \mathbf{A}_{b}^{\mathrm{ns}} & \mathbf{C}_{1}^{\mathrm{ns}} & \mathbf{C}_{2}^{\mathrm{ns}} & \cdots & \mathbf{C}_{s}^{\mathrm{ns}}
\end{array}\right]\left\{\begin{array}{c}
\mathbf{x}_{b}^{(n), 1} \\
\mathbf{x}_{b}^{(n), 2} \\
\vdots \\
\mathbf{x}_{b}^{(n), \mathrm{ns}} \\
\mathbf{x}_{1}^{(n)} \\
\mathbf{x}_{2}^{(n)} \\
\vdots \\
\mathbf{x}_{s}^{(n)}
\end{array}\right\}} \\
& =\left[\begin{array}{cccc}
\mathbf{B}_{\bar{b}}^{1} & & & \\
& \mathbf{B}_{\bar{b}}^{2} & & \\
& & \ddots & \\
& & & \mathbf{B}_{\bar{b}}^{\tilde{n} \mathrm{~s}}
\end{array}\right]\left\{\begin{array}{c}
\mathbf{y}_{\bar{b}}^{(n), 1} \\
\mathbf{y}_{\bar{b}}^{(n), 2} \\
\vdots \\
\mathbf{y}_{\bar{b}}^{(n), \mathrm{ns}}
\end{array}\right\}+\left\{\begin{array}{c}
\mathbf{r}_{\bar{b}}^{(n), 1} \\
\mathbf{r}_{\bar{b}}^{(n), 2} \\
\vdots \\
\mathbf{r}_{\bar{b}}^{(n), \mathrm{ns}}
\end{array}\right\},
\end{aligned}
$$

where $n$ is the current time step, $s$ is the number of interfaces, $\mathbf{C}_{i}^{k}$ denotes the coupling submatrix associated with the interface $\Gamma_{i}$ and the $k$ th subregion (containing terms of $\mathbf{H}_{i}^{k}$ and $\mathbf{G}_{i}^{k}$ ), and $\mathbf{x}_{i}^{(n), k}$ and $\mathbf{y}^{(n), k}$ are the vectors containing the unknown and prescribed values at the $n$th time point, respectively. It should be noticed that the system (27) represents the most general form of a coupled system. Indeed, for most of the problems, some of the coupling matrices $\mathbf{C}_{i}^{k}$ are null; particularly when the subdomains are coupled in a chain way, like for instance finite element models for beams, the coupled system (27) reduces to a banded form [12]. In the algorithm proposed in this paper, the system (27) is actually not assembled; instead one works directly with the subsystems (25), as they were uncoupled. The use of iterative methods makes it possible to solve the system of Eq. (27) in an implicit way. To see how to do this, we first give the general expressions of the bi-conjugate gradient algorithm (BiCG method) [11].

Iterative formula:

$\mathbf{x}^{n+1}=\mathbf{x}^{n}+\lambda_{n} \mathbf{p}^{n}$.

Search directions:

$\mathbf{p}^{n}= \begin{cases}\mathbf{r}^{0}, & \text { if } n=0, \\ \mathbf{r}^{n}+\alpha_{n} \mathbf{p}^{n-1}, & \text { if } n \geq 1,\end{cases}$
${ }^{*} p^{n}= \begin{cases}{ }^{*} \mathbf{r}^{0}=\mathbf{r}^{0}, & \text { if } n=0, \\ { }^{*} \mathbf{r}^{n}+\alpha_{n}{ }^{*} \mathbf{p}^{n-1}, & \text { if } n \geq 1 .\end{cases}$ 


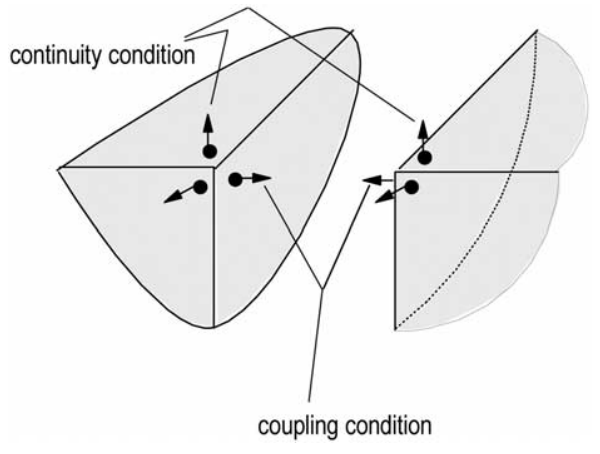

Fig. 3. Coupling and continuity conditions.

Residuals:

$\mathbf{r}^{n}=\mathbf{r}^{n-1}-\lambda_{n-1} \mathbf{A} \mathbf{p}^{n-1}$,

${ }^{*} \mathbf{r}^{n}={ }^{*} \mathbf{r}^{n-1}-\lambda_{n-1} \mathbf{A}^{\mathrm{T} *} \mathbf{p}^{n-1}$.

Parameters:

$\lambda_{n-1}=\frac{{ }^{*} \mathbf{r}^{n-1, \mathrm{~T}} \mathbf{r}^{n-1}}{{ }^{*} \mathbf{p}^{n-1, \mathrm{~T}} \mathbf{A} \mathbf{p}^{n-1}}, \quad \alpha_{n}=\frac{{ }^{*} \mathbf{r}^{n, \mathrm{~T}} \mathbf{r}^{n}}{{ }^{*} \mathbf{r}^{n-1, \mathrm{~T}} \mathbf{r}^{n-1}}$.

Two points are observed in the above iterative formulae: (1) the only operations necessary are multiplications matrixvector and vector-vector; (2) the system matrix is not transformed during its resolution (as usual in iterative procedures). So, a special solution procedure can be developed to solve the system (27) in which the corresponding coupled system is not explicitly assembled. In effect, a loop over the number of all subregions is carried out and the contribution of each subregion, independently of each other, is taken into account in the operation considered (matrixvector or vector-vector). Naturally, in suitable points of the solution algorithm (solver) the data of each subdomain are updated, so as to satisfy the coupling and, if necessary, also the flux continuity conditions at the interface nodes. At the end, the final operation results of the current iteration are obtained by gathering the corresponding partial ones.

At nodes pertaining to interfaces between subregions $i$ and $j$, i.e. if $\mathbf{x} \in \Gamma_{i j}$, the coupling conditions are given by

$\left\{\begin{array}{l}u^{i}(\mathbf{x}, t)=u^{j}(\mathbf{x}, t) \\ p^{i}(\mathbf{x}, t)=-p^{j}(\mathbf{x}, t)\end{array}, \quad\right.$ if $\mathbf{n}^{i}(\mathbf{x})=-\mathbf{n}^{j}(\mathbf{x})$,

and the continuity ones, by

$$
\left\{\begin{array}{l}
u^{i}(\mathbf{x}, t)=u^{j}(\mathbf{x}, t) \\
p^{i}(\mathbf{x}, t)=p^{j}(\mathbf{x}, t)
\end{array}, \quad \text { if } \mathbf{n}^{i}(\mathbf{x})=\mathbf{n}^{j}(\mathbf{x}) .\right.
$$

In Fig. 3, pairs of interface nodes are shown in which the coupling and continuity conditions should be considered.

In the computer code, a research is carried out in order to identify the interfaces (through their nodes) and then to introduce automatically the coupling and, if necessary, continuity conditions.

The interfaces between the subregions are defined by sets of coupled nodes, which are in turn defined based on the following criterion: coupled nodes pertain to two different domains, have the same co-ordinates, and opposite unit normal outward vectors. Associated with coupled nodes that have not the same geometrical position as those of other interfaces, that is, for non-common interface nodes (Fig. 4), the respective number of equations is always enough, without any additional consideration on the interface variables, to calculate the potential and normal flux at them. In this case one will obtain, for each node, two equations for determining one potential and one flux. In case of common interface nodes (Fig. 4), that is, if the coupled node is common to two or more interfaces, some additional considerations on the interface variables must be introduced in order to reduce the number of the corresponding unknowns; namely, flux continuity conditions must be taken into account (see Eq. (34) and Fig. 3).

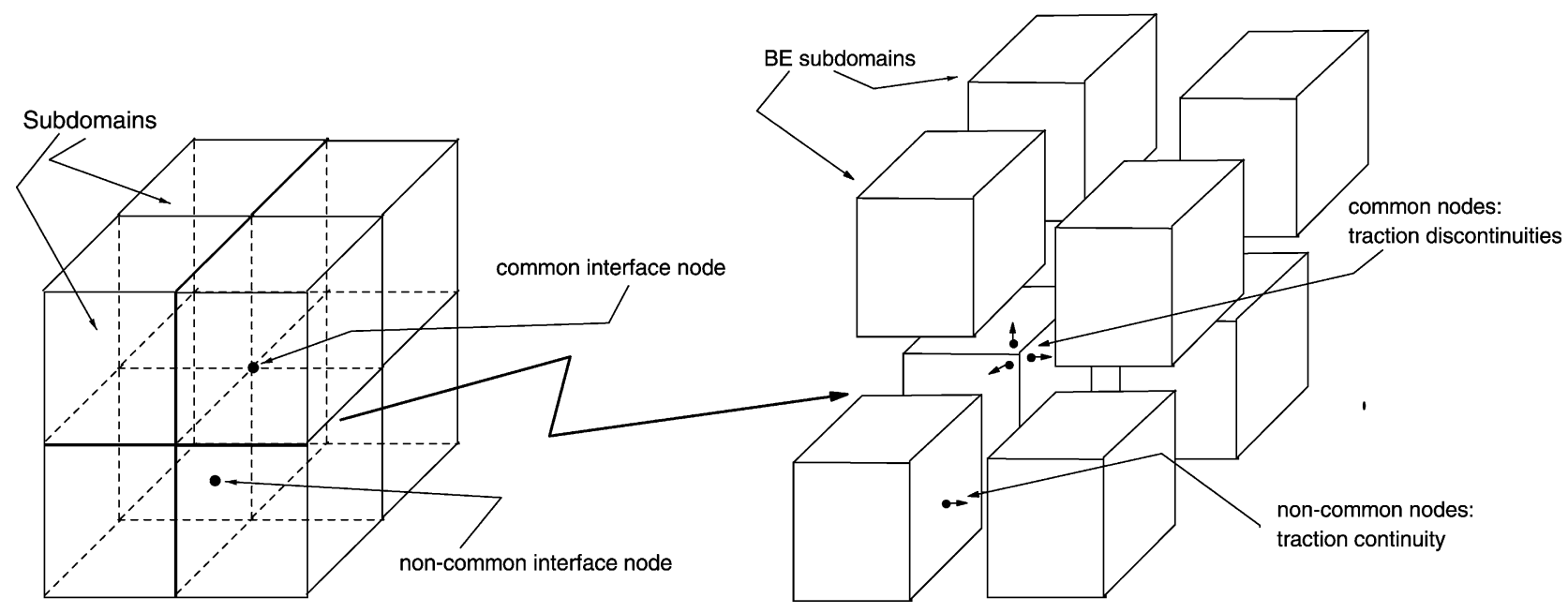

Fig. 4. Coupled subdomains and interface nodes (common and non-common ones). 


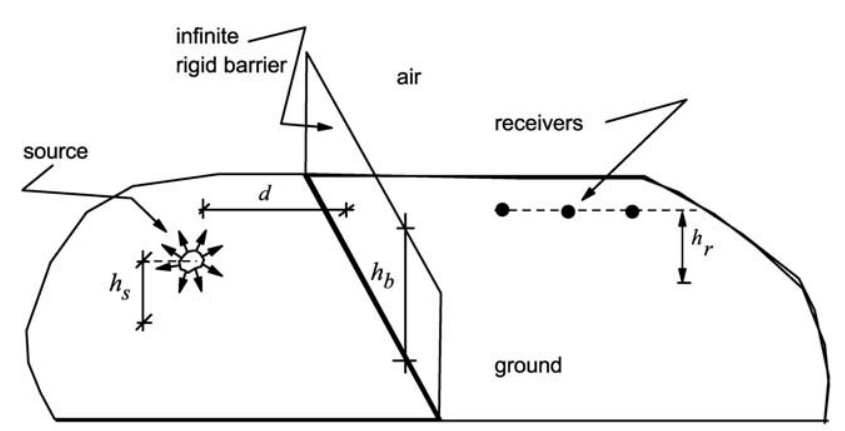

Fig. 5. Infinite sound barrier.

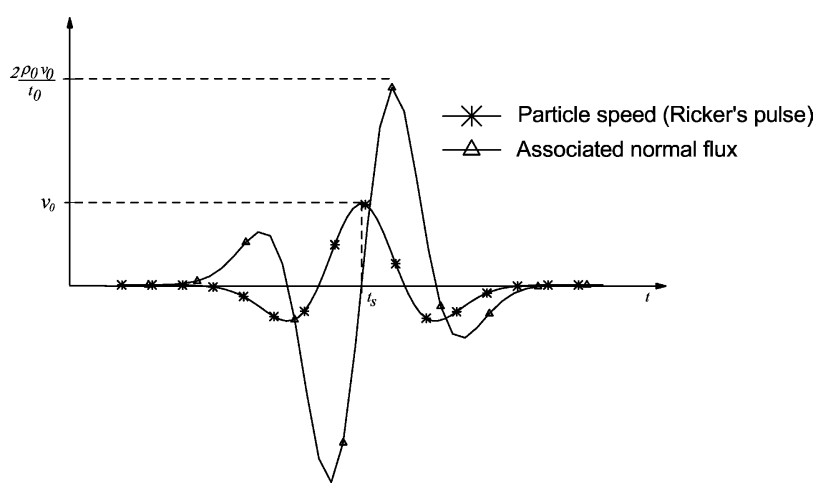

Fig. 6. Ricker's pulse.

\section{Applications}

In order to observe the performance of the algorithm, the sound pressure distribution caused by an acoustic energy source, in the absence and presence of an infinite nonthickness barrier of height $h_{\mathrm{b}}=4.0 \mathrm{~m}$, is analyzed (Fig. 5). The acoustic waves are radiated by a pulsating surface located at height $h_{\mathrm{s}}=1.5 \mathrm{~m}$, and at distance $d=5.0 \mathrm{~m}$ from the barrier. The temporal variation of the normal component of the particle velocity is given by following pulses:

\subsection{Sinusoidal pulse}

In this case, the particle velocities are given by

$v_{n}(\mathbf{x}, t)=v_{0} \sin (\omega t)$.

The corresponding normal fluxes are

$p(\mathbf{x}, t)=-\rho_{0} v_{0} \omega \cos (\omega t)$.

For the analysis one considered $v_{0}=5.00 \mathrm{~m} / \mathrm{s}$ and an excitation frequency corresponding to the octave-band center frequency $f=63 \mathrm{~Hz}$.

\subsection{Ricker's pulse}

Here, the speed of the particles and corresponding flux are expressed by (Fig. 6)

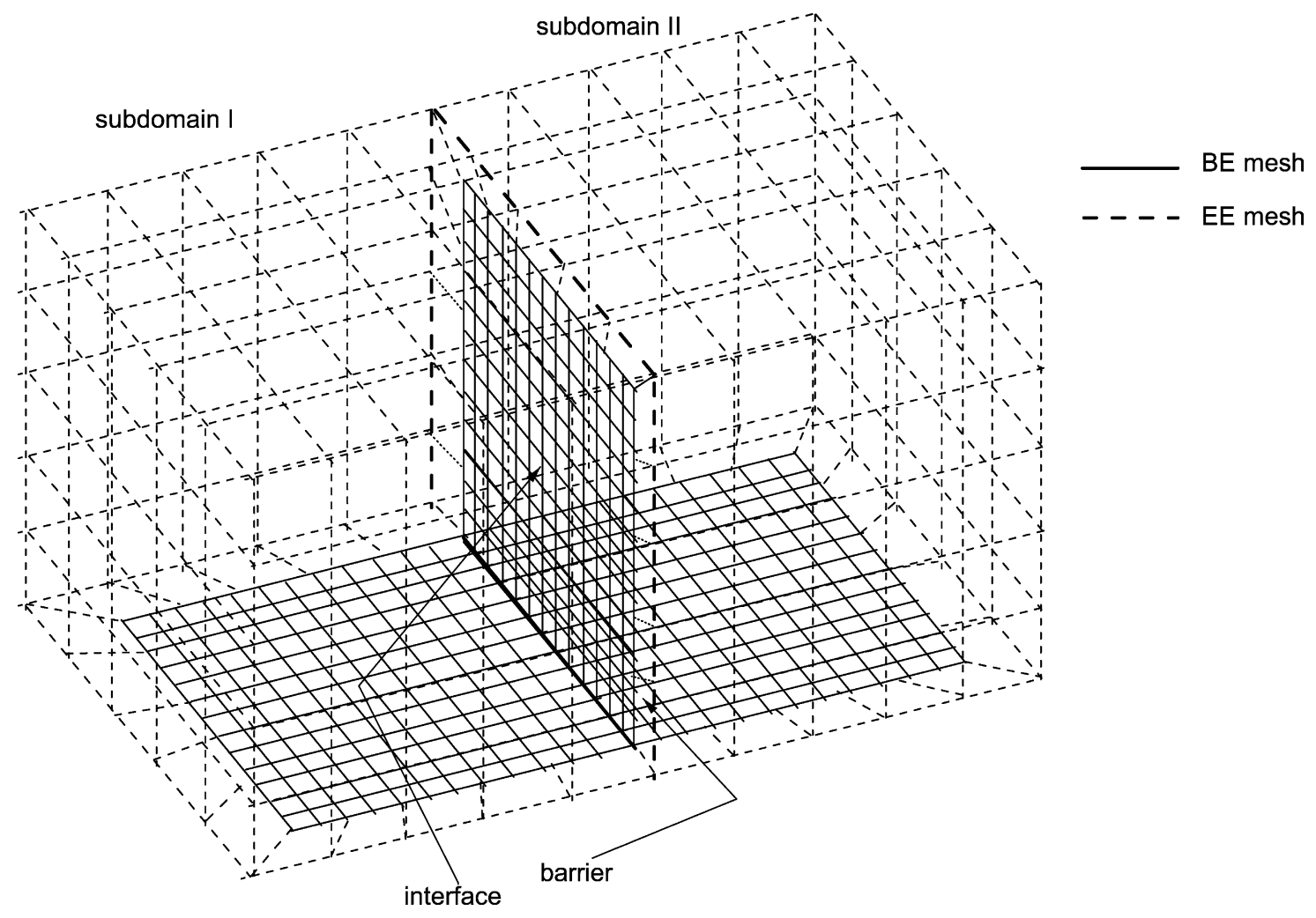

Fig. 7. Mesh of boundary and enclosing elements. 


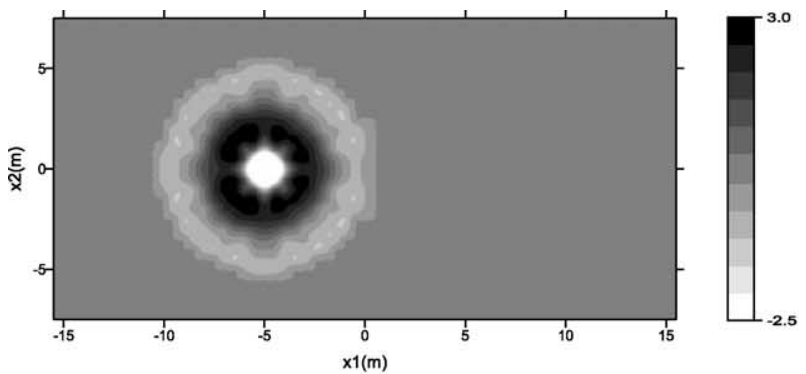

$t=0.015 s$

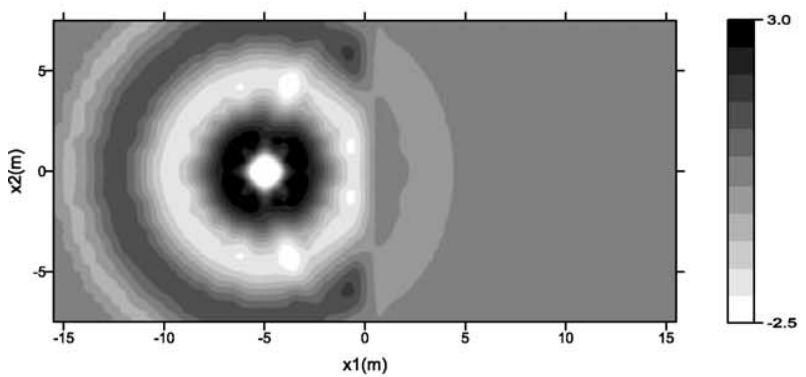

$t=0.030 \mathrm{~s}$
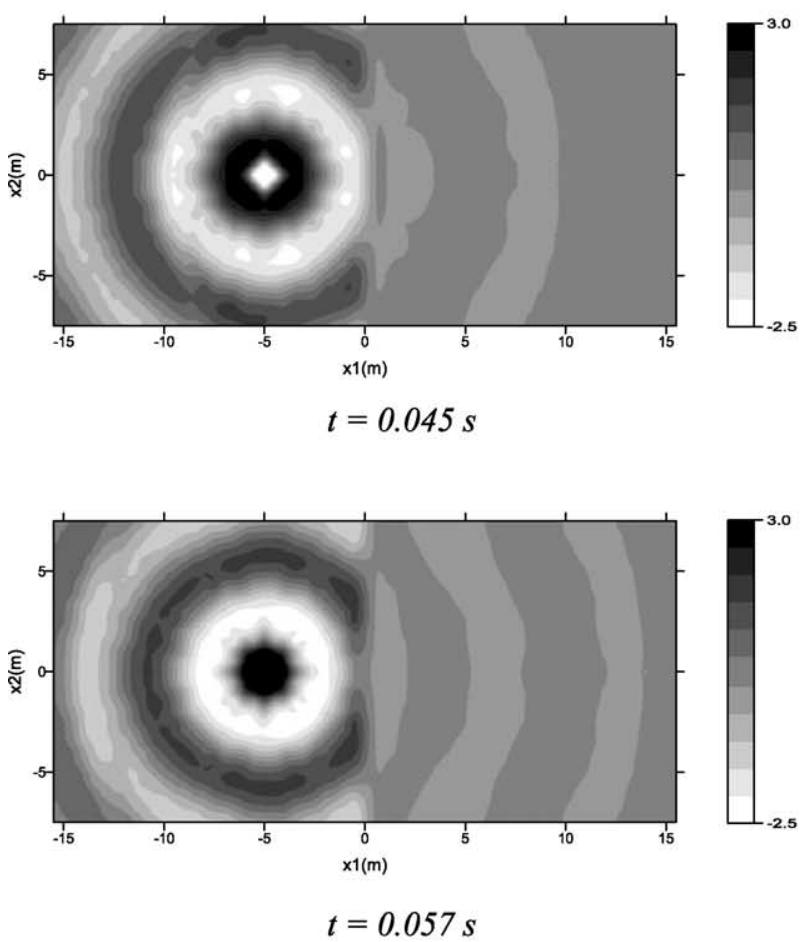

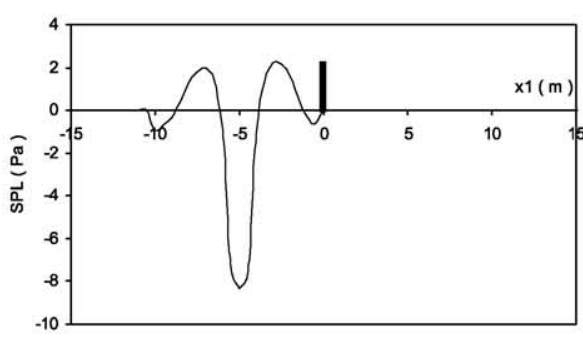

Sectional view $\left(x_{2}=0.0 \mathrm{~m}, t=0.015 \mathrm{~s}\right)$

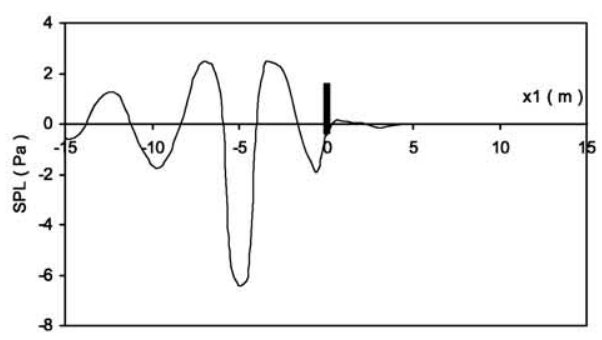

Sectional view $\left(x_{2}=0.0 \mathrm{~m}, t=0.030 \mathrm{~s}\right)$

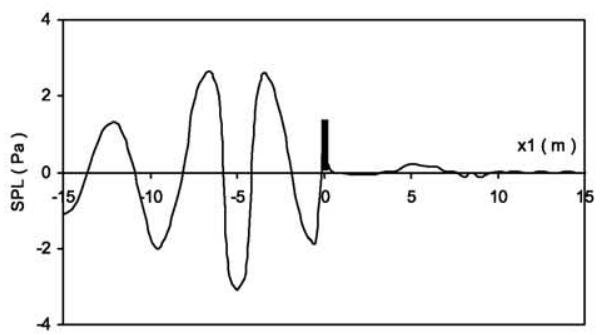

Sectional view $\left(x_{2}=0.0 \mathrm{~m}, t=0.045 \mathrm{~s}\right)$

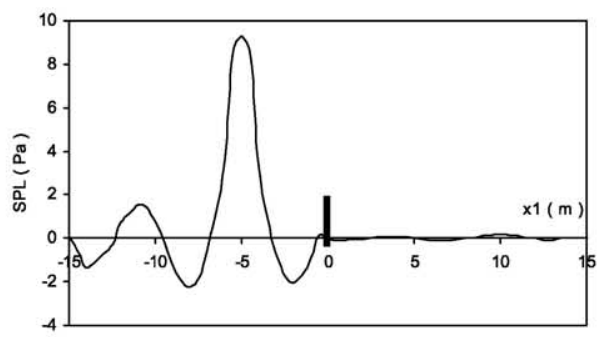

Sectional view $\left(x_{2}=0.0 \mathrm{~m}, t=0.057 \mathrm{~s}\right)$

Fig. 8. Time-domain response (sinusoidal pulse).

$p(\mathbf{x}, \tau)=-\frac{\rho_{0} v_{0}}{t_{0}}\left(4 \tau^{3}-6 \tau\right) \mathrm{e}^{-\tau^{2}}$

where $\tau=\left(t-t_{\mathrm{s}} / t_{0}\right), t$ represents the time, $t_{\mathrm{s}}$ is the time point corresponding to the pulse amplitude, and $\pi t_{0}$, the dominant wavelet period [23]. A velocity amplitude of $v_{0}=$
$5.00 \mathrm{~m} / \mathrm{s}$, and a dominant period of $\pi t_{0}=(1 / 63) \mathrm{s}$ were considered.

The source was tridimensionally simulated as a sphere of radius $a=0.05 \mathrm{~m}$. As the dimensions of the source are much smaller than the length of the acoustic wave generated $(\lambda=5.397 \mathrm{~m})$, the details of the pulsating surface will not indeed affect the sound being radiated. 
The boundary element mesh (Fig. 7) is generated so that the element sides be approximately $1 / 4$ of the wavelength. Eight-noded (parabolic) boundary elements are adopted, and two subregions are considered to model the barrier. Additionally a mesh of enclosing elements is used to make it possible to implicitly evaluate the principal values in the open subdomains. The BE mesh for subregion I has 1060 nodes and 320 elements, and for subregion II, 962 nodes and 288 elements. The respective EE meshes (of enclosing elements) for both
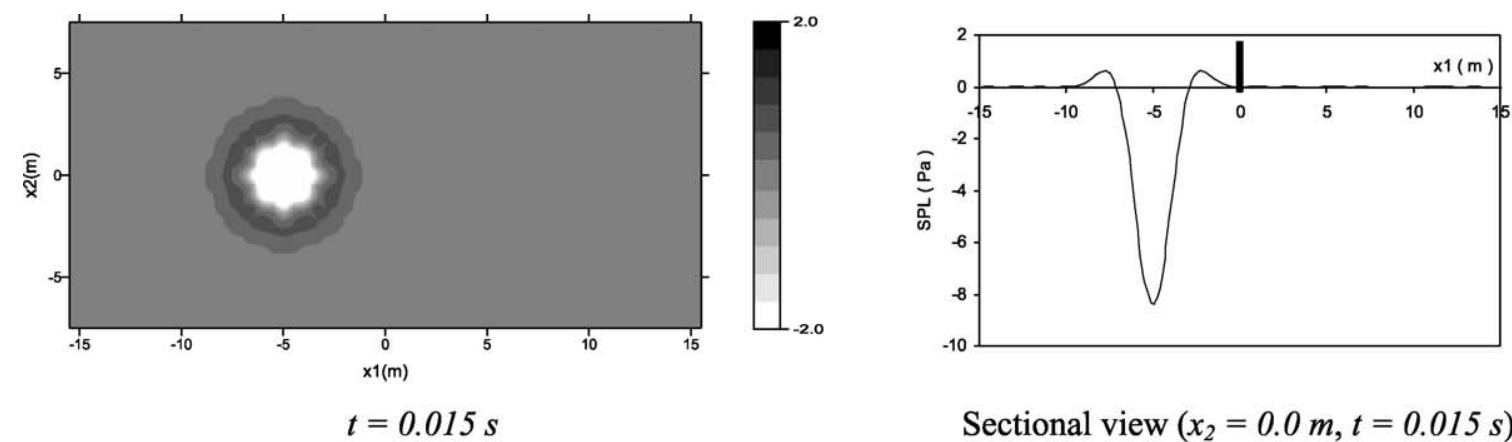

Sectional view $\left(x_{2}=0.0 \mathrm{~m}, t=0.015 \mathrm{~s}\right)$
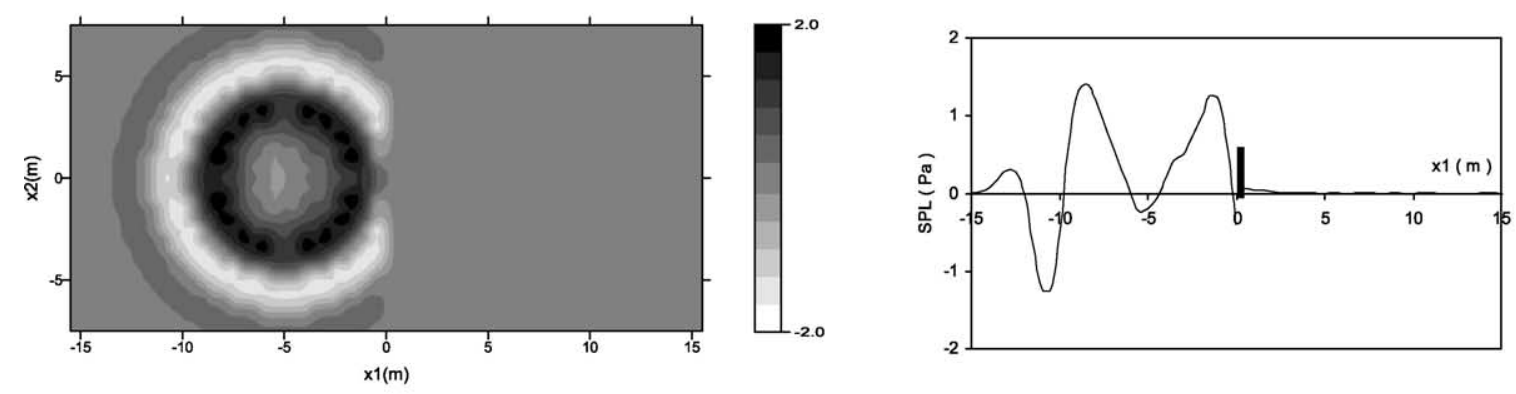

$t=0.030 s$

Sectional view $\left(x_{2}=0.0 m, t=0.030 \mathrm{~s}\right)$
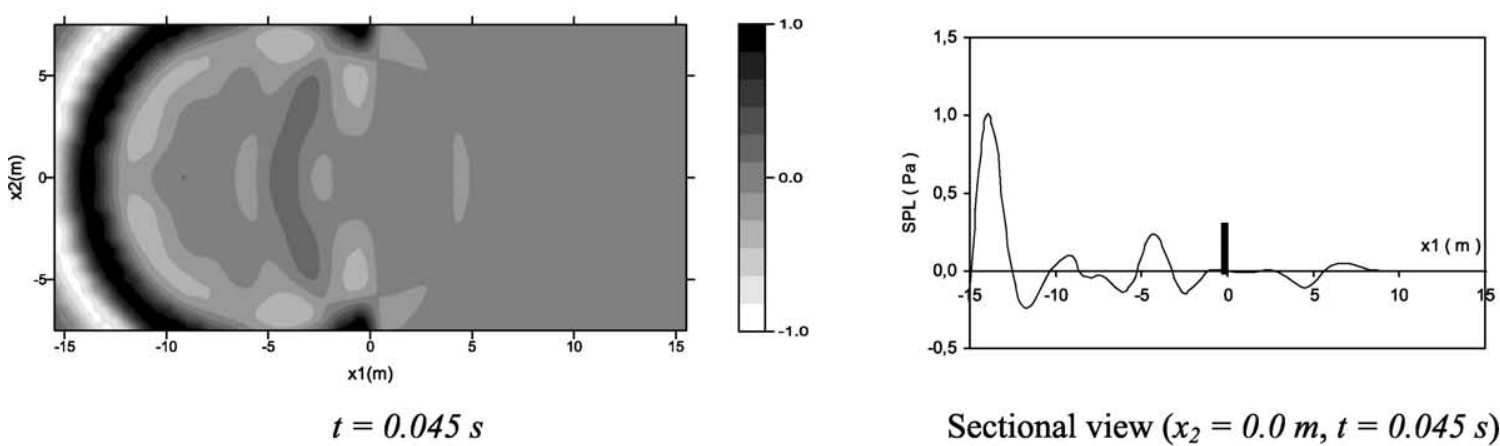

Sectional view $\left(x_{2}=0.0 \mathrm{~m}, t=0.045 \mathrm{~s}\right)$
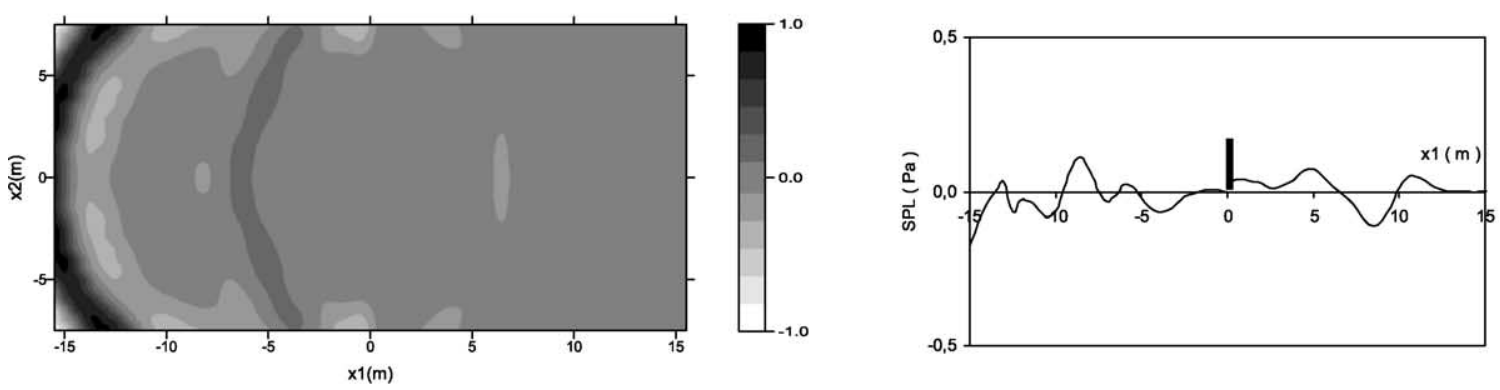

$t=0.057 \mathrm{~s}$

Sectional view $\left(x_{2}=0.0 \mathrm{~m}, t=0.057 \mathrm{~s}\right)$

Fig. 9. Time-domain response (Ricker's pulse). 
subdomains have 421 nodes and 130 elements. The source (spherical shell of radius $a=0.05 \mathrm{~m}$ ) is modeled with 32 boundary elements; it is located in the subregion 1 and is not visible in Fig. 7. The physical constants of the considered medium (air) are:

$c=340.00 \mathrm{~m} / \mathrm{s} \quad$ (sound speed),

$\rho_{0}=1.21 \mathrm{~kg} / \mathrm{m}^{3} \quad$ (equilibrium density).

The adopted time step, $\Delta t$, is determined by observing the wave period $T$ and the $\beta$ coefficient, the latter being defined through

$\beta=\frac{c \Delta t}{d}$

where $d$ is the minimal diagonal of all elements of the $\mathrm{BE}$ mesh. The following criterion is adopted to choose the time step: it should not be too long compared with the wave period $T$, and the corresponding $\beta$ coefficient, not so different from the unity. For the analyses here, $\Delta t=1.5 \times 10^{-3} \mathrm{~s}$ was chosen, which corresponds approximately to one-tenth of the wave period and to a $\beta=0.27$.

The results in terms of sound pressure level (SPL) are shown, for certain time points, in Figs. 8 and 9 for the sinusoidal pulse and the Ricker's pulse, respectively. The sequence of snapshots in these figures displays the acoustic wave propagation in the presence of the barrier. The snapshots obtained in the absence of the barrier are not displayed.

The response in terms of insertion loss (IL) for both pulses is shown in Fig. 10. The receiver points, at which the IL is calculated, are located behind the barrier at height $h_{\mathrm{r}}=1.5 \mathrm{~m}$ above the ground (Fig. 5), and the following

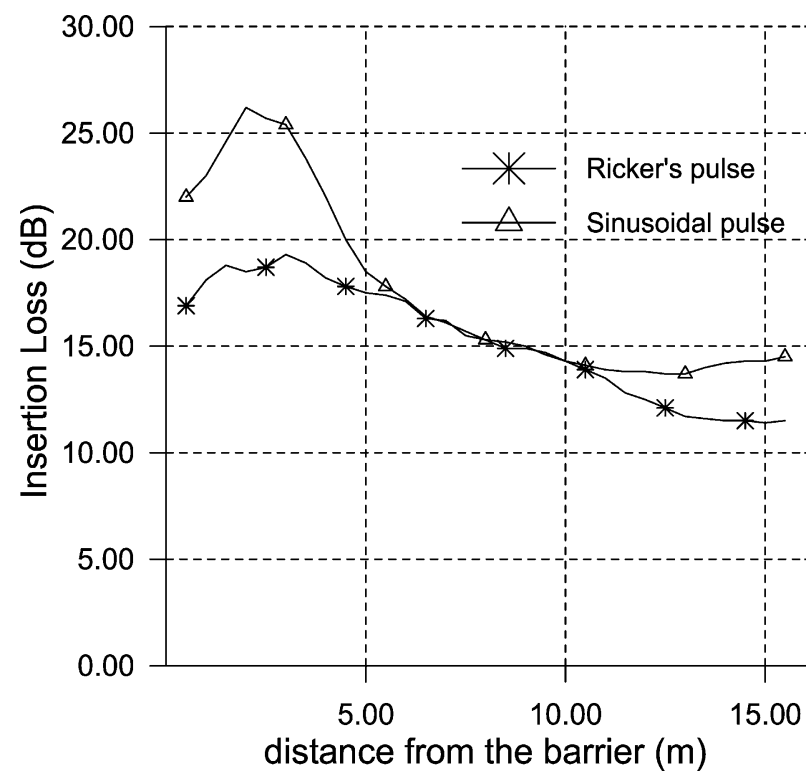

Fig. 10. Insertion loss curve.

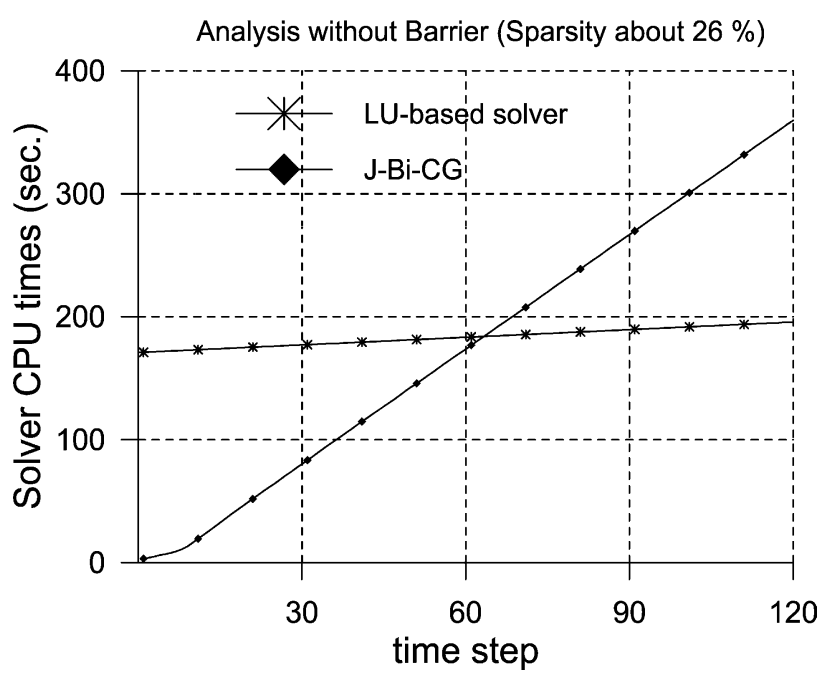

Fig. 11. CPU time curve (without barrier).

expression is used to evaluate the insertion loss (in $\mathrm{dB}$ )

$\mathrm{IL}(\mathrm{dB})=-20 \log \frac{u_{\mathrm{b}}}{u_{\mathrm{f}}}$,

where $u_{\mathrm{b}}$ and $u_{\mathrm{f}}$ are the acoustic pressures in the presence and absence of the barrier, respectively.

For the analyses carried out in this paper, the $\mathrm{BE} / \mathrm{BE}$ coupling strategy based on the Jacobi-preconditioned biconjugate gradient solver was used [11]. To give an idea of the performance of this coupling strategy, the CPU times attained in the analysis are plotted against the sequence of time steps (Figs. 11 and 12). The direct solver considered for comparison is based on the non-symmetric LU decomposition and is non-optimized as regards the treatment of the matrix sparsity, which for the analyses here corresponds to approximately 26 and 32\%, without and with barrier, respectively.

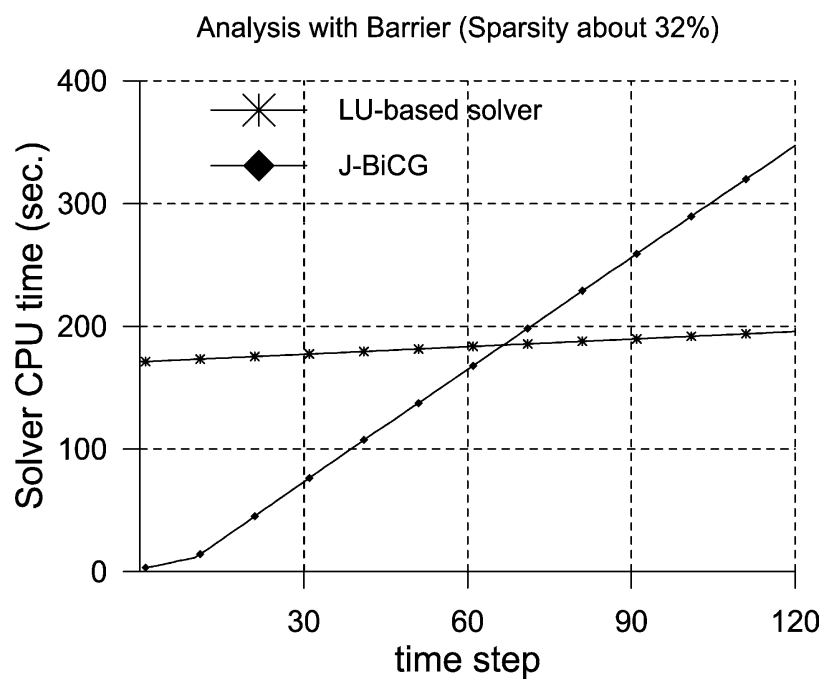

Fig. 12. CPU time curve (with barrier). 
The iterative scheme was stopped whenever $\left\|\mathbf{r}^{n+1}\right\| \leq$ tol; $\mathbf{r}^{n+1}$ is the norm of the residual vector at the current iteration and tol $=10^{-5}$, the adopted tolerance.

The computer used for carrying out the analyses had an AMD ATHLON processor with $1.2 \mathrm{GHz}$ and 768 Mbytes random access memory. The code was developed in FORTRAN and ran in a LINUX environment.

\section{Conclusions}

With the aid of the graphics in Figs. 8 and 9, which show the response in terms of SPL for both pulses considered, in the presence of an infinite barrier, the effectiveness attained by the barrier in reducing the noise in the acoustic shadow area $\left(x_{1}>0.00 \mathrm{~m}\right)$ is visualized. Details of the time evolution of the wave propagation phenomenon, including superposition of incident and scattered waves, can also be observed.

More information on the barrier effectiveness can be taken from the insertion loss curves given in Fig. 10. As one can see, the barrier is more effective for those points closer to it. The position of the receivers at which maximum efficiency is found can also be directly determined from the IL curves for both pulses.

The information related to the computational effectiveness of the algorithm is presented in Figs. 11 and 12, which show the CPU time values against the time steps. As expected, for the first time steps, the coupling strategy based on the iterative solver is much more effective than the LU-based one because the J-Bi-CG scheme is much faster than the LU-based solver. However, as a time-dependent linear problem is treated, the latter coupling algorithm begins to be more effective than the former when the number of time steps increases (after the 60th time step, see Figs. 11 and 12). Despite this (expected) fact, it should be noticed that the coupling strategy presented in this paper, which works directly with the subsystems and does not assemble the global matrix, may be a very interesting analysis tool for large industrial applications, mainly 3D ones. In such cases a LU decomposition may be a very hard computational task.

The frequency of the acoustic source studied is actually low; for higher frequencies the meshes must be suitably refined. Thus, the numerical effort for carrying out the time-domain analysis may increase considerably. Nevertheless, the strategy is general, and may be applied to any complex time-dependent problems described by the acoustic theory considered in his paper. Furthermore, the strategy is very suitable for developing parallelized computer codes. On the other hand, for nonlinear time-dependent problems, the presented strategy is, for sure, quite convenient.

\section{References}

[1] Kinsler LE, Frey AR, Coppens AB, Sanders JV, Fundamentals of acoustics, New York: Wiley; 1982.

[2] Mansur WJ, Brebbia CA. Numerical implementation of the boundary element method for two-dimensional transient scalar wave propagation problems. Appl Math Modelling 1982;6:299-306.

[3] Mansur WJ, Brebbia CA. Formulation of the boundary element method for transient problems governed by the scalar wave equation. Appl Math Modelling 1982;6:307-11.

[4] Mansur WJ. A time-stepping technique to solve wave propagation problems using the boundary element method. PhD Thesis. Southampton University, Southampton, England; 1983

[5] Dohner JL, Shoureski R, Bernhard RJ. Transient analysis of three dimensional wave propagation using the boundary element method. Int J Numer Meth Engng 1987;24:621-34.

[6] Meise T. BEM calculation of scalar wave propagation in 3-D frequency and time domain (in German). PhD Thesis. Fakultät für Bauingenieurwesen, Ruhr-Universität, Bochum, Germany; 1990

[7] Antes H. Applications in environmental noise. In: Ciskowiski RD, Brebbia CA, editors. Boundary element methods in acoustics. New York: Computational Mechanics Publications; 1991. p. 225-60.

[8] Araújo FC, Mansur WJ, Carrer JAM. Time-domain three dimensional analysis. In: Wu TW, editor. Boundary element acoustics. Fundamentals and computer codes. London: Computational Mechanics Publications; 2000. p. 159-216.

[9] Antes H, Baaran J. Noise radiation from moving surfaces. Engng Anal Bound Elem 2001;25:725-40.

[10] Gennaretti M, Morino L. A boundary element method for the potential, compressible aerodynamics bodies in arbitrary motion. Aeronaut J R Aeronaut Soc 1992.

[11] Araújo FC, Martins CJ, Mansur WJ. An efficient BE iterative-solverbased sub-structuring algorithm for 3D time-harmonic problems in elastodynamics. Engng Anal Bound Elem 2001;25:795-803.

[12] Cremers L, Fyfe KR, Sas P. A variable order infinite element for multi-domain boundary element modeling of acoustic radiation and scattering. Appl Acoust 2000;59:185-220.

[13] Banerjee PK. The boundary element methods in engineering. London: McGraw-Hill; 1994.

[14] Bialecki RA, Merkel M, Mews H, Kuhn G. In- and out-of-core BEM equation solver with parallel and non-linear options. Int J Numer Meth Engng 1996;39:4215-42.

[15] Krishnasamy G, Rizzo FJ, Liu Y. Boundary integral equations for thin bodies. Int J Numer Meth Engng 1994;37:107-21.

[16] de Lacerda LA, Wrobel LC, Mansur WJ. A dual boundary element formulation for sound propagation around barriers over an impedance plane. J Sound Vib 1997;202(2):235-47.

[17] de Lacerda LA, Wrobel LC, Power H, Mansur WJ. A novel boundary integral formulation for three-dimensional analysis of thin acoustic barriers over an impedance plane. J Acoust Soc Am 1998;104(2):671-9.

[18] de Lacerda LA, Wrobel LC, Power H, Silva JJR, Mansur WJ. Analysis of thin acoustic barriers using slender-body theory. Boundary elements in acoustics. Advances and applications, 1st ed. Southampton: Computational Mechanics Publications; 2000.

[19] Rosen D, Cormack DE. The continuation approach for singular and near singular integration. Engng Anal Bound Elem 1994;13:99-113.

[20] Mantic V. On computing boundary limiting values of boundary integrals with strongly singular and hypersingular kernels in 3D BEM for elastostatics. Engng Anal Bound Elem 1994;13:115-34.

[21] Kawai T, Hidaka T, Nakajima T. Sound propagation above an impedance boundary. J Sound Vib 1982;83:125-38.

[22] Chandler-Wilde SN, Hothersall DC. Sound propagation above an inhomogeneous impedance plane. J Sound Vib 1985;98:475-91.

[23] Godinho L, António J, Tadeu A. Sound propagation around rigid barriers laterally confined by tall buildings. Appl Acoust 2002;63: $595-609$. 
[24] Rigby RH, Alliabadi MH. Out-of-core solver for large, multi-zone boundary element matrices. Int J Numer Meth Engng 1995;38: 1507-33.

[25] Ganguly S, Layton JB, Balakrishna C, Kane JH. A fully symmetric multi-zone Galerkin boundary element method. Int J Numer Meth Engng 1999;44:991-1009.

[26] Axelsson O. Iterative solution methods. New York: Cambridge University Press; 1994.

[27] Hackbusch W. Iterative solution of large linear systems of equations. New York: Springer; 1994.

[28] Saad Y. Iterative methods for sparse linear systems. New York: PWS Publishing; 1996.

[29] Mansur WJ, Araújo FC, Malaghini JEB. Solution of BEM systems of equations via iterative techniques. Int J Numer Meth Engng 1992;33: 1823-41.

[30] Barra LPS, Coutinho ALGA, Telles JCF, Mansur WJ. Multi-level hierarchical pre-conditioners for boundary element systems. Engng Anal Bound Elem 1993;12:103-9.
[31] Prasad KG, Kane JH, Keyes DE, Balakrishna C. Preconditioned Krylov solvers for BEA. Int J Numer Meth Engng 1994;37: 1651-72.

[32] Hribersek M, Skerget L. Iterative methods in solving Navier-Stokes equations by the boundary element method. Int J Numer Meth Engng 1996;39:115-39.

[33] Leung CY, Walker SP. Iterative solution of large three-dimensional BEM elastostatic analyses using the GMRES technique. Int J Numer Meth Engng 1997;40:2227-36.

[34] Skerget L, Hribersek M, Kuhn G. Computational fluid dynamics by boundary domain integral method. Int J Numer Meth Engng 1999;46: $1291-311$.

[35] Valente FP, Pina HL. Iterative techniques for 3-D boundary element method systems of equations. Engng Anal Bound Elem 2001;25: $423-9$.

[36] Sleijpen GLG, van der Vorst HA. An over-view of approaches for the stable computation of hybrid BiCG methods. Appl Numer Math 1995; 19:235-54. 